\title{
Rainbow glare after laser-assisted in situ keratomileusis: a review of literature
}

\author{
This article was published in the following Dove Press journal: \\ Clinical Ophthalmology \\ 9 November 2016 \\ Number of times this article has been viewed
}

\section{Majid Moshirfar ${ }^{1,2}$ \\ Jordan D Desautels ${ }^{3}$ \\ Tyler S Quist ${ }^{4}$ \\ David F Skanchy ${ }^{5}$ \\ Mark T Williams ${ }^{6}$ \\ Ryan T Wallace ${ }^{7}$}

'Department of Ophthalmology and Visual Sciences, John A Moran Eye Center, University of Utah School of Medicine, Salt Lake City, UT, ${ }^{2} \mathrm{HDR}$ Research Center, Hoopes Vision, Draper, UT, ${ }^{3}$ Tufts University School of Medicine, Boston, MA, ${ }^{4}$ University of Utah School of Medicine, Salt Lake City, UT, ${ }^{5}$ McGovern Medical School, The University of Texas Health Science Center at Houston, Houston, TX, 'University of California, San Francisco School of Medicine, San Francisco, CA, ${ }^{7}$ Brigham Young University, Provo, UT, USA
Correspondence: Majid Moshirfar HDR Research Center - Hoopes Vision, I 820 S State Street Suite \#200,

Draper, UT 84020, USA

$\mathrm{Tel}+\mathrm{I} 8015680200$

$\mathrm{Fax}+\mathrm{I} 8015630200$

Email cornea2020@me.com

\begin{abstract}
This article reviews the current literature pertaining to rainbow glare ( $R G)$, including incidence rate, clinical presentation, etiology, prognosis, and management. RG is a rare optical complication of femtosecond laser-assisted in situ keratomileusis that results in patients seeing an array of spectral bands surrounding point sources of light under mesopic and scotopic conditions. The mechanism is thought to be a consequence of the formation of a transmissive diffraction grating on the posterior surface of the corneal flap created by the FS laser. RG has a good prognosis and is usually self-limiting. Persistent RG with concomitant residual refractive error may warrant lifting the flap and photoablating the posterior surface of the flap. Patients with persistent RG and no residual refractive error should be considered candidates for phototherapeutic keratectomy on the posterior flap surface.
\end{abstract}

Keywords: rainbow glare, femtosecond, LASIK, keratomileusis, phototherapeutic keratectomy

\section{Introduction}

Femtosecond laser-assisted in situ keratomileusis (FS-LASIK) surgery performed using FS lasers for corneal flap creation has become increasingly safe and effective over time. ${ }^{1}$ However, several characteristic complications of FS-LASIK, including transient light sensitivity syndrome, problematic bubble formation, and optical side effects such as rainbow glare (RG), remain clinically important. ${ }^{1} \mathrm{RG}$ is a unique complication, in that it is intimately tied to the quality of the laser beam and the spot pattern generated by the beam at the flap interface. $\mathrm{RG}$ describes a condition where patients observe spectral bands emanating symmetrically from point sources of light under dark background conditions. ${ }^{2}$ This review aims to summarize the current literature pertaining to RG, comment on the current state and future of RG incidence, and provide recommendations for surgeons facing $\mathrm{RG}$ in the clinic.

\section{Incidence and epidemiology}

$\mathrm{RG}$ was first described in the literature by Krueger et al in $2008 .{ }^{2}$ In this report, the overall incidence of RG was found to be $19.14 \%$ for eyes treated with an older model of IntraLase (Abbott Medical Optics Inc, Santa Ana, CA, USA) 15 kHz FS laser (pulse energy, 1.3-2.6 $\mu \mathrm{J}$; spot/line separation, 9/9-11/9 $\mu \mathrm{m}$ ) and $2.47 \%$ for eyes treated with a newer, higher numerical aperture model of the same $15 \mathrm{kHz}$ laser (pulse energy, $1.3 \mu \mathrm{J}$; spot/line separation, 10/9 $\mu \mathrm{m}$ ). The incidence of RG was higher among hyperopic eyes (39.4\%) than myopic eyes $(14.8 \%)$ in the group of patients treated with the older laser model. Further, an increased degree of myopic correction was associated with an increased incidence of RG. The depth of the flap interface was also an important determinant of $\mathrm{RG}$ occurrence, with a $19 \%$ increase in 
incidence per additional $10 \mu \mathrm{m}$ of interface depth. The only postoperative aberrometry values found to be significantly associated with increased RG were coma (12\% increase in incidence per $0.1 \mu \mathrm{m}$ increase at 3 months) and total higher-order aberrations ( $9 \%$ increase per $0.1 \mu \mathrm{m}$ increase at 3 months). Preoperative corneal pachymetry has not been found to be associated with RG. RG was also significantly associated with other postoperative symptoms such as halos, glare, and starbursts. ${ }^{2}$

A subsequent study in 2009 by Bamba et al investigated the incidence of RG in 256 patients who underwent LASIK surgery with a $60 \mathrm{kHz}$ IntraLase FS laser (pulse energy, $0.8 \mu \mathrm{J}$ [ $75 \%$ of eyes] and $1.0-1.1 \mu \mathrm{J}$ [ $25 \%$ of eyes]; spot separation, $8 \mu \mathrm{m}) .{ }^{3}$ The overall incidence of RG was found to be $5.8 \%$, with a higher incidence observed in the $1.0-1.1 \mu \mathrm{J}$ group $(11.6 \%)$ than in the $0.8 \mu \mathrm{J}$ group (4.1\%). However, even with the increased frequency, decreased raster energy, and smaller spot separation employed by Bamba et al, ${ }^{3}$ the incidence rate in their study did not decline below $2.47 \%$, which was observed by Krueger et al when using comparatively less optimal surgical settings. It was also shown that the duration of time between scheduled laser maintenance visits is correlated to the incidence of RG. ${ }^{2,3}$ Interestingly, this cohort showed no relationship between RG incidence and refractive error. Other reports have also described the occurrence of RG using a $60 \mathrm{kHz}$ IntraLase laser., ${ }^{4,5}$

RG has also been observed in surgeries performed with higher frequency FS lasers such as the FS-200 Wavelight laser (Alcon Laboratories, Inc., Fort Worth, TX, USA)., ${ }^{4,6}$ It has been shown that the incidence rate of RG following procedures performed with the FS-200 laser is not significantly different from procedures performed with the IntraLase $60 \mathrm{kHz}$ laser. ${ }^{4}$ No cases of RG have been reported with the IntraLase $150 \mathrm{kHz}$ system or VisuMax (Carl Zeiss Meditec AG, Jena, Germany). There have also been no reported cases after small-incision lenticule extraction. Overall, the incidence of RG does not appear to be correlated with age or sex. ${ }^{3}$ None of the studies have evaluated any other epidemiological risk factors such as iris color.

\section{Clinical presentation and etiology}

A majority of the patients who experience RG report their symptoms immediately after surgery. ${ }^{2,7}$ The appearance of the rainbow pattern varies slightly from patient to patient, but is generally described as 4-12 spectral bands radiating symmetrically from a point source of light. Dark conditions enhance the perception of RG, making nighttime driving problematic for many patients. ${ }^{2,3}$ In rare instances, patients report seeing continual halos of color rather than discrete bands. ${ }^{2}$ In all cases, the red component (longer wavelength) of each spectral band is observed to be more peripheral than the violet-blue component (shorter wavelength). RG is not accompanied by changes in visual acuity.

No overt slit lamp findings are associated with RG; however, confocal microscopy has clearly demonstrated the presence of a grid-like pattern of hyperreflective spots corresponding to the raster pattern of the FS laser. ${ }^{6,7}$ This raster grid pattern is thought to be the cause of RG; however, this pattern is also observable in patients without symptoms of RG. ${ }^{8}$

With regards to etiology, RG is thought to be a consequence of the formation of a transmissive diffraction grating on the posterior surface of the corneal flap by the FS laser. ${ }^{2}$ In general, when polychromatic light is shone onto a grating pattern with regularly spaced slits, a spectral pattern comprising monochromatic beams is created as light diffracts through each slit and constructively interferes on the other side. The angle of emergence of each spectral component for a given mode of diffraction $(\mathrm{m}=0,1,2, \ldots)$ is directly related to its wavelength $(\lambda)$ and inversely related to the distance between the centers of each slit $(A)$ by the following diffraction grating equation: $\sin \theta_{\mathrm{m}}=\mathrm{m} \lambda / A$ (Figure 1). Thus, blue wavelengths appear closer to the light source and red wavelengths appear further away.

Analogously, when a raster pattern of periodically spaced laser impacts is produced on the posterior surface of a corneal flap, it creates a grating pattern that can act as a diffractive medium capable of producing a spectral pattern. In confirmation of this notion, Krueger et al estimated that a grating pattern with a spot spacing (A) of $9.22 \mu \mathrm{m}$ would produce spectral bands with emergence angles $\left(\theta_{\mathrm{m}}\right)$ consistent with those reported by their patients using the methodology outlined by them (Figure 2). ${ }^{2}$ This theoretical spacing of $9.22 \mu \mathrm{m}$ was nearly identical to the programmed spot spacing of the FS laser $(\sim 9 \mu \mathrm{m})$.

Owing to the inverse relationship between the emergence angle of the spectral bands and the spot spacing of the laser, it may seem that reducing the spot/line separation could increase the angle of the spectral pattern sufficient enough to avoid photodetection by the retina altogether. While a theoretical minimum spot/line spacing to avoid RG does exist, this approach to avoiding RG is clinically impractical because the required spot/line separation is far below what is achievable by current systems. For example, even a spot/line separation of $1 \mu \mathrm{m}$ would produce an emergence angle of $\sim 24^{\circ}$, which over the axial length of an average eye $(\sim 23 \mathrm{~mm})$ would strike 


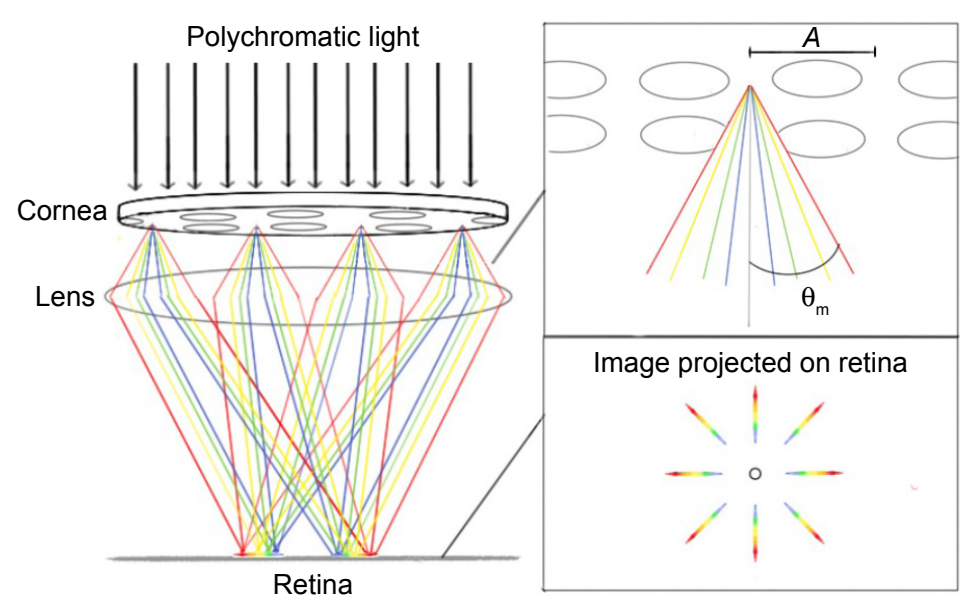

Figure I Schematic of rainbow glare.

Notes: The centers of slits created by laser spots on the cornea during flap creation are separated by a distance, $A$, and diffract light of wavelength, $\lambda$, at an emergence angle, $\theta_{m}$, for a given mode of diffraction $(m=0,1,2, \ldots)$. The distance between the centers of consecutive slits is related to the diffraction angle by the following diffraction grating equation: $A=\mathrm{m} \lambda / \sin \theta \mathrm{m}$. The fundamental mode of diffraction $(\mathrm{ml})$ represents the brightest set of bands and is therefore the most clinically significant.

the retina well within the area of photodetection as the density of color-sensitive cones would still be adequate for perception of rainbow color. Thus, it should be noted that even smaller values of spot/line separation (ie, $4 / 4$ or $2 / 2 \mu \mathrm{m}$ ) employed by newer FS laser systems are still capable of producing $\mathrm{RG}$ when the beam is poorly focused and compromises the smoothness of the stromal bed.

The diffraction grating hypothesis has also been supported by several modeling studies. After first noting the RG effect, Krueger et al irradiated a glass slide with the causative IntraLase laser and observed a spectral pattern similar to patterns reported by their patients. ${ }^{2}$ Similar RG effects have been observed after FS irradiation of test gels and contact lenses. ${ }^{3,9}$ A more comprehensive study that investigated the optical side effects of different FS flap creation patterns (raster, spiral, and random) on model corneas showed that only a random pattern was capable of entirely preventing the occurrence of RG. ${ }^{10}$ Current FS laser systems are not capable of producing a randomized beam pattern for flap creation. Excimer lasers used to photoablate the stroma are capable of producing randomized patterns, yielding a possible explanation for why RG is exclusively described as resulting from the posterior surface of the flap rather than the stromal bed.

Furthermore, RG has never been reported when using a spiral flap creation pattern in real patients. Irrespective of whether this finding is a result of a true decline in incidence or decreased reporting, it is important to recognize that a spiral pattern still has the necessary spot periodicity to produce RG. ${ }^{10}$ Thus, the apparent decline in RG when using a spiral pattern should not be attributed to the change in pattern from raster to spiral, but rather to better quality optics that reduce the formation of a roughly grated flap surface. Until the FS laser systems are capable of creating flaps using

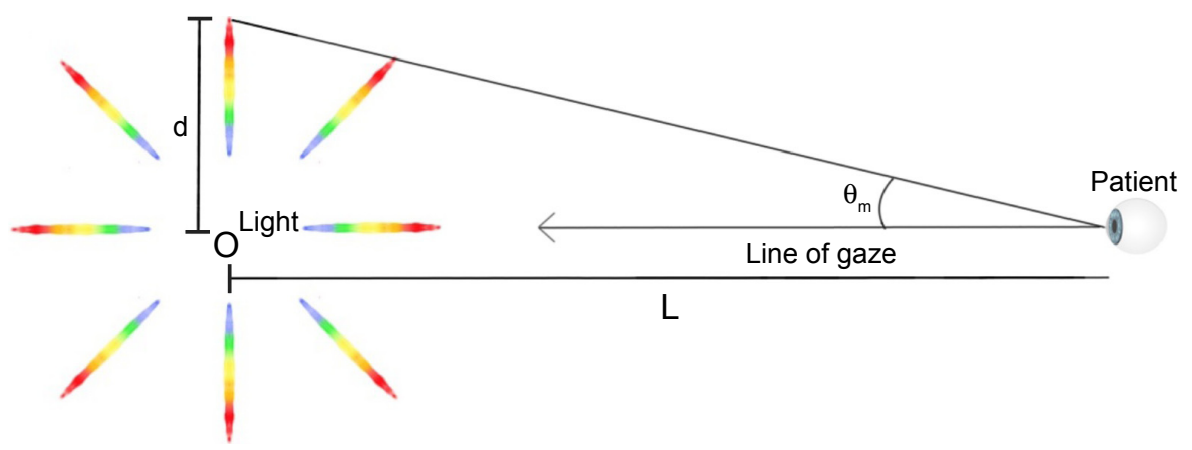

Figure 2 Mathematical method for calculating the theoretical slit spacing to be compared to the programmed spot/line spacing on the femtolaser laser outlined by Krueger et al. ${ }^{2}$

Notes: A point of light source is pushed through a piece of white paper which is held at arms' length. The patient can draw the spectrum they see on the piece of paper and the corresponding angle, $\theta_{m}$, can be calculated. Knowing the angle, it is easy to calculate the theoretical spot spacing using the diffraction grating equation. 
a randomized shot pattern, the best strategy for preventing $\mathrm{RG}$ remains maximizing the numerical aperture of the laser objective and regularly maintaining the system to ensure optimal beam alignment.

Given the importance of beam management in the prevention of $\mathrm{RG}$, it is useful to consider the mechanism by which decreased numerical aperture or poor beam alignment could determine the occurrence of RG. The three-dimensional shape of the laser spots created by the FS laser on the posterior surface of the corneal flap has not been described in detail. However, it is reasonable to conclude that a beam with a larger focal spot volume will increase the dimensions of the impact crater created by the laser on the posterior flap surface. The increased focal spot volume imparted by low numerical aperture and poor beam alignment may extend the depth of the laser impact crater beyond the plane of the flap interface, creating a roughly grated surface. Conversely, a tightly focused beam from a high-numerical aperture objective lens will create laser spots that are more confined to the focal plane and more likely to be smoothed over by the plasma explosion of adjacent spots during flap creation. This is congruent with the finding that RG incidence tends to increase as a function of time elapsed between scheduled maintenances of the laser system. ${ }^{2,3}$

In addition to the physical dimensions of each spot, the material that fills in the crater created by each spot could alter the diffractive properties of the grating pattern. It is currently unknown whether each crater is filled in by interstitial fluid, gas, or another material after its formation. ${ }^{10}$ Because different materials possess varying refractive qualities, the residual material in the craters is perhaps important for enhancing the effect of RG. Although the most logical fill material is interstitial fluid, overly energetic and/or poorly focused beams could result in small pockets of residual gas remaining in the impact craters. The subsequent absorption of this air could possibly explain the early transience of some RG cases.

Although most patient-described RG patterns are adequately explained by a diffraction grating created by the FS laser during flap creation, there are other possible mechanisms that may cause RG. Theoretically, any process that generates regularly spaced slits in light-transmitting tissues of the eye is capable of generating a diffraction pattern that could be perceived as RG. One such possible mechanism is the creation of a regular chatter pattern at the flap interface after rapid gas escape during flap creation using an FS laser. Interestingly, $\mathrm{RG}$ has not been reported with the use of microkeratomes for flap creation. Despite the ability of microkeratomes to generate chatter patterns as a potential complication at the flap interface, these patterns are quite irregular in nature and do not serve as viable diffraction gratings to create $\mathrm{RG}$.

\section{Prognosis and physician guidelines}

$\mathrm{RG}$ is often a transient process with most patients experiencing complete resolution of symptoms by the ninth postoperative month. However, there is no consensus on the treatment of persistent RG. Gatinel et al experienced success with photoablation of the posterior surface of the flap during a simultaneous correction for astigmatism. ${ }^{6,11}$ In the absence of refractive error, the benefit of lifting the flap may present unnecessary cost and risk to the patient. Cases of persistent RG, which causes significant distress to emmetropic patients, can be treated using a 6-15 $\mu \mathrm{m}$ phototherapeutic keratectomy (PTK) on the back surface of the flap. Excimer tracking of the pupil cannot be accomplished on the posterior surface of the flap. Thus, the center of the flap should be marked prior to reflection. Any hyperopia induced by the PTK can be corrected with a compensatory photorefractive keratectomy (PRK) on the epithelial side of the flap. The best strategy for the overall management of RG is still prevention, and surgeons should strictly adhere to routinely scheduled laser maintenance to keep beam parameters optimal.

\section{Conclusion and future directions}

$\mathrm{RG}$ is a rare complication of FS-LASIK surgery. The incidence of this complication has decreased due to continual technological improvements that have optimized the focal properties of FS lasers used to create corneal flaps. Despite these improvements, $R G$ is still a potential optical side effect of FS-LASIK, particularly in the setting of poorly managed laser systems. Patients generally present with symptoms of RG immediately after surgery and describe the spectral pattern as multiple rainbow bands radiating from point sources of light in dark conditions. Their vision is otherwise unaffected. Mechanistically, this condition is thought to result from the formation of a diffraction grating on the posterior flap surface by the FS laser. The overall prognosis for this condition is good; however, surgical corrective options exist for cases of persistent RG. Management using PRK or PTK of the posterior surface of the flap should be decided based on the degree of residual refractive error following LASIK. The primary preventative measure should be proper laser system maintenance. Future work should focus on the incidence rate and explore mechanisms to prevent the occurrence of $R G$ using more modern FS lasers. 


\section{Acknowledgment}

This work was supported in part by an unrestricted grant from

Research to Prevent Blindness, New York, New York.

\section{Disclosure}

The authors report no conflicts of interest in this work.

\section{References}

1. Stonecipher KG, Ignacio TS, Stonecipher KG, Thompson V. Management of Complications in Refractive Surgery: Femtosecond Laser. Berlin Heidelberg: Springer; 2008.

2. Krueger RR, Thornton IL, Xu M, Bor Z, van den Berg TJ. Rainbow glare as an optical side effect of IntraLASIK. Ophthalmology. 2008;115(7): 1187-1195.

3. Bamba S, Rocha KM, Ramos-Esteban JC, Krueger RR. Incidence of rainbow glare after laser in situ keratomileusis flap creation with a $60 \mathrm{kHz}$ femtosecond laser. J Cataract Refract Surg. 2009;35(6):1082-1086.

4. Krueger RR, Rocha KM, Dupps Jr WJ. Natural History and Incidence of Rainbow Glare and Light Scattering in Femtosecond LASIK. Paper 16815. San Diego, CA: American Society of Cataract and Refractive Surgery; 2015.
5. Salz JJ. Difficult and Complicated Cases in Refractive Surgery: Suction Loss After Complete Raster Pattern and No Side Cut, No Flap Lift, and Rainbow Glare. Berlin Heidelberg: Springer; 2014.

6. Gatinel D, Saad A, Guilbert E, Rouger H. Unilateral rainbow glare after uncomplicated femto-LASIK using the FS-200 femtosecond laser. J Refract Surg. 2013;29(7):498-501.

7. Gatinel D, Guilbert E, Saad A. Rainbow Glare After FemtosecondLASIK: Lessons Learned From 5 Consecutive Cases. San Diego, CA: American Society of Cataract and Refractive Surgery; 2015.

8. Sonigo B, Iordanidou V, Chong-Sit D, et al. In vivo corneal confocal microscopy comparison of intralase femtosecond laser and mechanical microkeratome for laser in situ keratomileusis. Invest Ophthalmol Vis Sci. 2006;47(7):2803-2811.

9. Peter M, Kammel R, Ackermann R, et al. Analysis of optical side-effects of fs-laser therapy in human presbyopic lens simulated with modified contact lenses. Graefes Arch Clin Exp Ophthalmol. 2012;250(12): 1813-1825.

10. Ackermann R, Kammel R, Merker M, Kamm A, Tünnermann A, Nolte S. Optical side-effects of fs-laser treatment in refractive surgery investigated by means of a model eye. Biomed Opt Expres. 2013;4(2):220-229.

11. Gatinel D, Saad A, Guilbert E, Rouger H. Simultaneous correction of unilateral rainbow glare and residual astigmatism by undersurface flap photoablation after femtosecond laser-assisted LASIK. J Refract Surg 2015;31(6):406-410.
Clinical Ophthalmology

\section{Publish your work in this journal}

Clinical Ophthalmology is an international, peer-reviewed journa covering all subspecialties within ophthalmology. Key topics include: Optometry; Visual science; Pharmacology and drug therapy in eye diseases; Basic Sciences; Primary and Secondary eye care; Patient Safety and Quality of Care Improvements. This journal is indexed on

Submit your manuscript here: http://www.dovepress.com/clinical-ophthalmology-journal

\section{Dovepress}

PubMed Central and CAS, and is the official journal of The Society of Clinical Ophthalmology (SCO). The manuscript management system is completely online and includes a very quick and fair peer-review system, which is all easy to use. Visit http://www.dovepress.com/ testimonials.php to read real quotes from published authors. 